\title{
Designing an Incapacitator Using LED
}

\author{
Burcu Yakışır Girgin ${ }^{1, *}$, Celal Zaim Çil ${ }^{2}$ \\ ${ }^{1}$ Nallıhan Vocational School, Ankara University, Turkey \\ ${ }^{2}$ Department of Electronic and Communication Engineering, Cankaya University, Turkey
}

Copyright $\mathrm{O} 2016$ by authors, all rights reserved. Authors agree that this article remains permanently open access under the terms of the Creative Commons Attribution License 4.0 International License

\begin{abstract}
An Incapacitator is a non-lethal device (NLD) that can be used by the security officers. We designed, developed an incapacitator using light emitting diodes (LEDs). We called this as blocker security system with LEDs (BSSL). Our objective to develop the BSSL is to test the hypothesis if the light pulses of varying intensity and color in time can cause incapacitation on human targets without permanent eye damages. We explained the design of the BSSL in detail, and discussed the effects on visual system and biological functions of the human being. We searched the best light pattern, if exists. We observed that the light patterns may indeed have effects especially when the frequency of light pulses are in the range of $7-15 \mathrm{~Hz}$, and the colors are blue, green, and red. Patterns at $15 \mathrm{~Hz}$ are especially effective as they coincide with the brain waves. To our knowledge, the LED incapacitators have not been investigated in this detail so far. The possibility of using such an NLD causing no permanent damage on the subjects is considered to be very important for the security forces.
\end{abstract}

Keywords Light Emitting Diode (LED), Incapacitator, Illuminance, Buck Driver, Human Visual System, Photopic Vision, Non-lethal Device

\section{Introduction}

Law enforcement officers and security services have always needed a tool that gives them a capability to incapacitate the subjects without causing a permanent injury and harm to them. To control and disperse a mob or stop a demonstration or seize an illegal border passers and refugees they need a device to make them incapacitate for a certain time. For these purposes they currently have been mostly using pepper gas, high-pressure water column, and when the situation gets worse even plastic bullets. Although these are not generally lethal they can cause serious injuries and even permanent disabilities on the people. Besides, such violent counter actions against people have a potential of generating deep hatred and enmities within and between masses and the security forces and mostly directed against governments with the powerful effects of the today's social and commercial media.

High-powered lasers or a very loud audio systems have been considered as alternatives to the above mentioned methods, and seldom used to incapacitate the people. However, lasers with a directed and coherent light beam can also give permanent damage to the eyes of the people, even blindness, if the beam hits the target's eye directly, and/or the power of the beam is not arranged properly. Similarly, a very loud sound can cause permanent damage to the ears of the people.

A very high intensity flashlight, not necessarily from a laser, may cause a temporary blindness in the subject. The person subjected to this light instinctively close his/her eyes or turn away, and this gives the security forces enough time to capture and take her/him under control. The research showed that light can affect a person mainly in two ways [1]. When a high intensity light is flashed at certain frequencies, called alpha frequencies of the brain at 8-12 Hz, people feel the flickering of the light the most and this can cause nausea, vomiting, disorientation and even temporary blindness, and sometimes vertigo. On the other hand when the color of the light is changing among red, green, and blue colors at certain frequency such that the person cannot focus herself in one color this causes headache, nausea, vomiting, confusion, disorientation, and visual impairment [1]. Utilizing these properties a non-lethal lamp can be made. The light source in such a lamp should be controlled precisely to arrange the frequency of the light pulses, to provide the intensity of the pulses, to form the light beam, and to provide the color of the consecutive light pulses as required.

Recently blue colored light emitting diodes (LEDs) have been developed with a very good efficacy (lumen per watt) levels and considerable optical power levels. The light from an LED is different from a laser in many ways. LED's light is diffuse, that is, the photons are not emitted in the same direction but in various directions, and having all the different phases. However in a laser, all the photons are emitted in the same direction, and have the same phase. The photons generated by the LEDs are of slightly different energies, colors, whereas in a laser they are essentially of the same energy (monochromatic). Due to these properties, light 
from a laser is formed in a very sharp beam when it emanates from the cavity of the laser. This means we have a very high energy of the beam within its small beam cross section. Therefore, when such a beam is directed to a person's eye it burns the cells in the retina and can cause the permanent blindness. However, LEDs cannot generate such a sharp beam of light, and generally are not so dangerous unless one's eyes get very close to the LED.

In parallel with the developments in the LEDs technology, the number of photons generated in a second (the light flux) have been increased to very high levels (such as 150 Lumens) recently [2]. This, together with the generation of white color from LEDs, make the LEDs a serious alternative to be used by illumination. If the powerful but incoherent light from a LED is directed and formed into a beam by using optical elements such as lenses and mirrors, achieving such a tool becomes possible without using laser. In addition, at a certain distance from the LED the intensity of the light (power per area) can be arranged such that a person can be incapacitated without causing a permanent harm. The colors generated by the LEDs cover a large spectrum ranging from ultraviolet, to visible and to infrared regions. Although the LEDs cannot produce a monochromatic light like a laser, they produce reasonably pure colors. Since the LEDs are semiconductor devices, they can be easily, and instantly switched on and off in nanoseconds [3]. Therefore, with their maturity and all of these advantages, the LEDs are good candidates to build very effective incapacitators.

Two LED Incapacitators have been reported in the literature so far, apart from the popular news appearing from time to time on the media. They both all belong to Rubstov [4, 5], where in the first one, registered in 2007, an array of relatively low-power LEDs are used to generate pulses spatially and temporally. In the second one, dated 2009 and updated in 2011, where the light pulses are not only generated temporally and spatially but also spectrally, by changing the color of the pulses. A range finder is also used in this incapacitator to arrange the light intensity at the target's eyes. In neither of the patents, the exact definition the patterns of the lights, that is, the temporal and spectral change of pulses was not given.

In this study we designed and developed a lamp as a prototype LED Incapacitator. We named this lamp as Blocker Security System with LED (BSSL). In the BSSL we used three LED chips, each containing four high power LEDs. Each LED on a chip emits different color, namely red, green, blue and white. Therefore, we have 12 LEDs in the BSSL altogether. We used four commercial off the shelf (COTS) buck LED drivers to drive the three same color LEDs in the three LED chips with a desired duty cycle arranged by the pulse width modulation (PWM) technique. We used four Metal-Oxide Semiconductor Field Effect
Transistor (MOSFET) switch circuits to modulate the light emitted in each color. MOSFET switches are controlled by a microcontroller. The modulation pattern that controls the frequency and the color of the light pulses emitted by the BSSL is managed by this microcontroller. We developed the software required. Therefore, the BSSL provides us a platform that can be used to search the best pattern of the light pulses that incapacitate a human target properly. The intensities of the pulses are arranged by the duty cycle of the buck drivers. We used lenses to shape the light beam of the BLLS.

In this paper we tested the hypothesis that if the human beings are subjected to the light pulses of proper intensities and colors in certain patterns in time they could be incapacitated. We give our design of the BSSL that can be used to generate the light pulses desired, and discuss how the effects of the light pulses changing temporally and spectrally cause incapacitation on a human target, and provide our findings regarding the light patterns that can produce the effects. In determining the effective light patterns we used ourselves as the subjects in the tests.

\section{Materials and Methods}

We decided to design and develop a prototype integrated lamp, the BSSL, which can be used to search the most effective light patterns to incapacitate the human beings within a range of 20 meters. The BSSL should provide a strobing mode that provides:

- temporal change of the intensities of the light pulses,

- spectral change of these pulses in many combinations of different color flashes,

- beam shaping (spatial configuration of light beam) of the light emitted.

- and a lantern mode (a flashlight) if needed when it is not used for incapacitation purposes.

The design of the BSSL should start with selecting the proper light sources, and the number of the light sources to be used within the integrated lamp together with the optical system to generate the beam of required intensity and shape, then the driver system to effectively provide the current and voltage to these light sources, and most importantly a practical way of changing the patterns of light in searching the effective patterns that produce the desired effects on the human target.

The design consists of the LED modules of required number, an optical system, a power supply to drive the LED module, an electronic control unit (signal circuit), and a mechanical system that provides housing for all these subsystems. Figure 1 a) shows our design concept for the BSSL, and b) shows the prototype developed. 

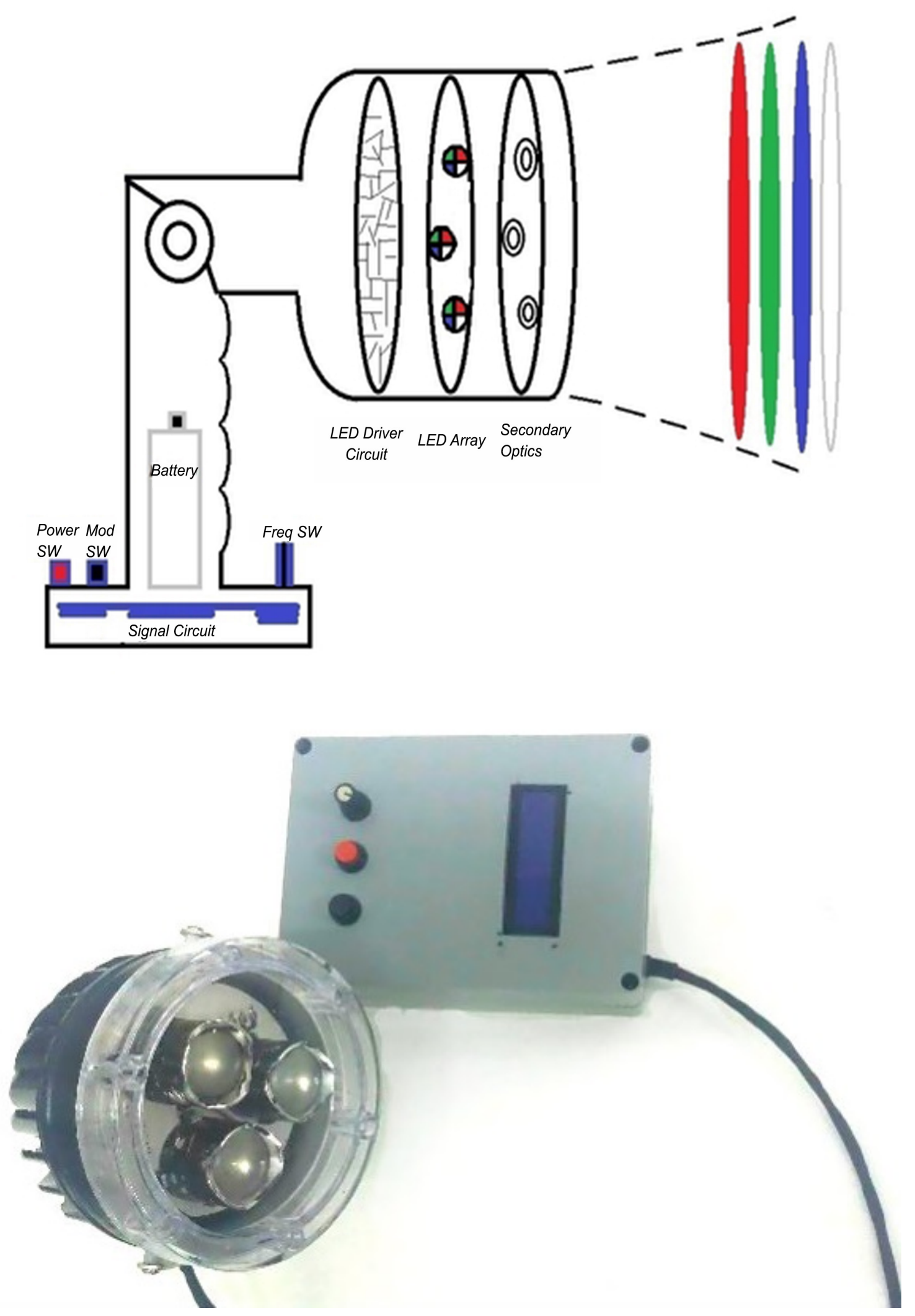

Figure 1. a) The basic design concept of the BSSL b) The BSSL Prototype 


\subsection{Selecting the Light Sources for the BSSL}

Our design of the BSSL started with the selection of the proper light source. We need light sources that can be easily switched on and off, having enough power to generate the light pulses of each primary colors: red, green, and blue (RGB) within the range. The lamp when used by the security forces should provide incapacitation of the target human beings, but in cases, it should also be used as a lantern too. For this purpose we need white light as well. White light can be produced by two ways: either by using blue light photons to generate white light through a phosphor layer; or by properly mixing the primary colors (RGB). We prefer the former method because in this way it is much easier to produce the light of white color with desired intensity.

To provide the colors and the intensity of the light and the switching properties needed, the LEDs with their present maturity technology are the best candidate for the light sources available. After this decision was made we have to select the type of the LEDs available on the market. LEDs to be used for illumination purposes are mostly introduced into the market in two forms: modules comprising of multiple LED chips bonded on a single board (Figure 2a), and modules comprising multiple single LED chips bonded on separate boards (Figure 2b).

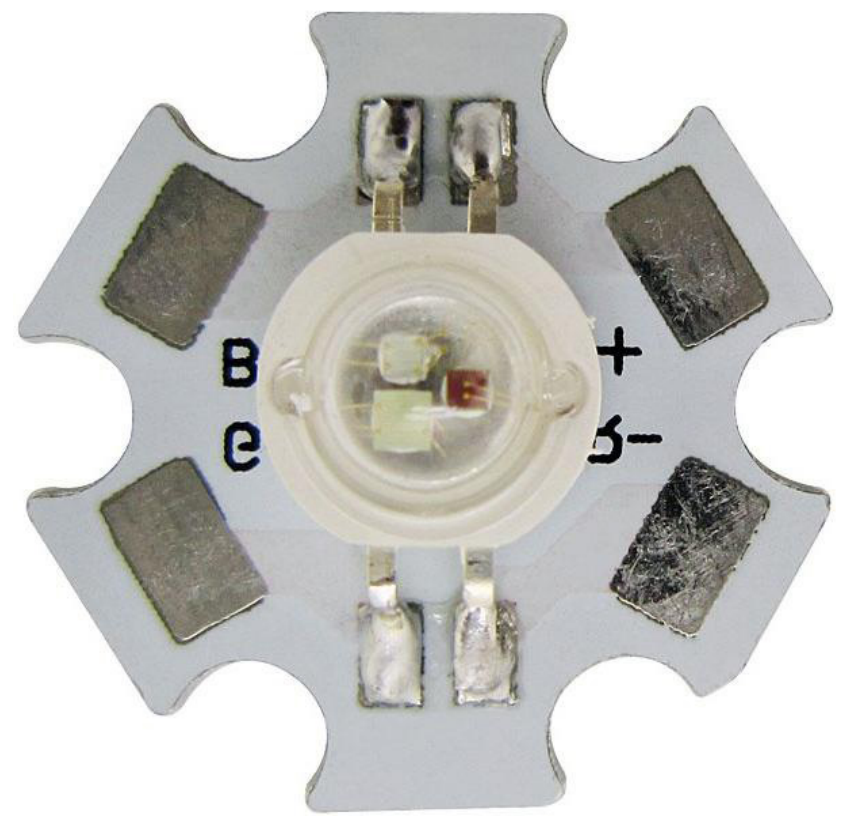

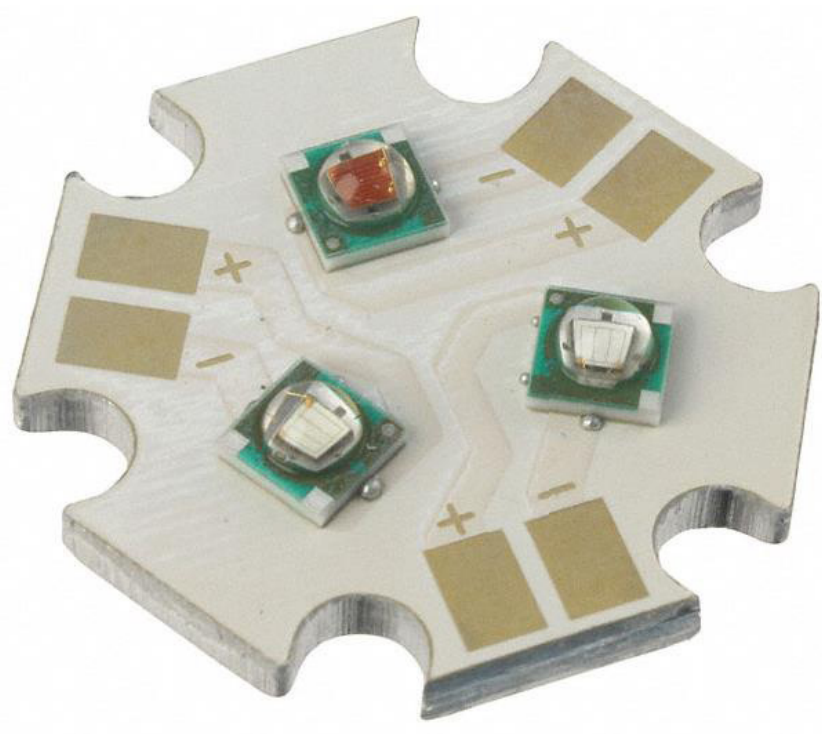

Figure 2. LED modules a) multiple LED chips (red, green, blue and amber LEDs) on a single board b) single LED chips on single boards (red, green, blue LEDs) (Edixeon), [6]

Since it comprises all primary colors (RGB), a LED module such as given in Figure 2 can be practically used in our BSSL design. The substrate that holds the LED chip(s) is aluminum and the LEDs are covered with an encapsulation material that optically shapes the light rays emitting from the semiconductor, and at the same time insulates the semiconductor from environmental disturbances such as dust, water vapor, finger prints. Aluminum substrate is providing a robust mechanical case and a very effective thermal conduction environment to dissipate the heat generated in the LED chip(s).

Since we desire a lantern mode also included in the BSSL a white light beam of desired intensity is needed, if this mode is selected by the user. LED module given in Figure 2a has a LED inside the chip that provides white light, whereas in LED module of Figure $2 b$ the RGB colors should be blended to obtain the white light. The latter method is not as effective in obtaining the white light of desired intensity as the former method. Additionally, focusing the light rays form the LEDs into a beam is much easier when we have all the LEDs on one chip than when we have LEDs on different chips. Therefore, we decided to use a module of LEDs as given in Figure 2a if it also provides the proper light intensities, beam shapes, and color qualities required.

We searched the market in May 2014 and decided to use a LED module of Edison Federal 5050 type as shown in Figure 3 , whose specifications are given in [7]. 


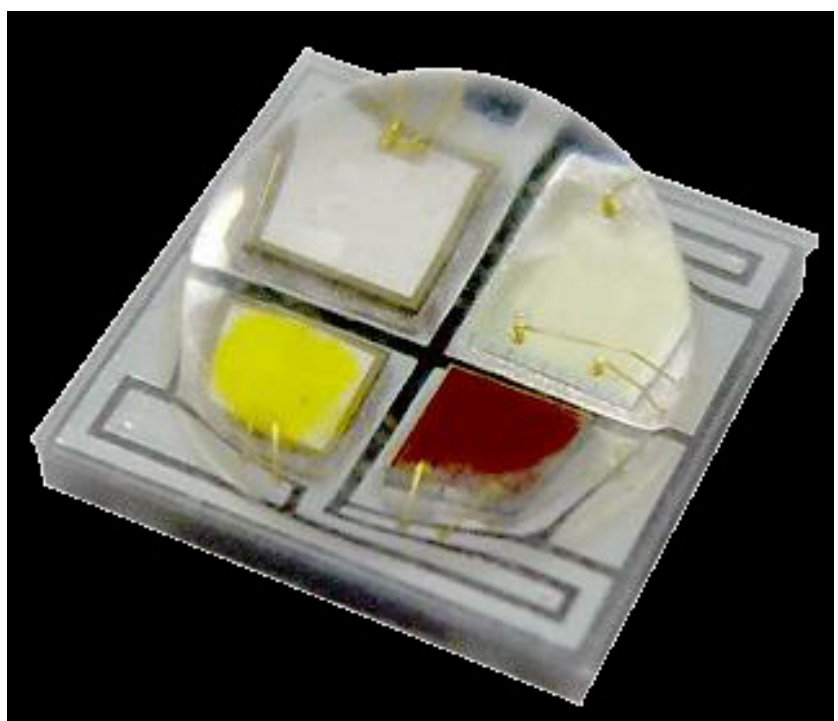

Figure 3. Edison Federal 5050 Type LED Module [7]
The luminous flux values and the forward voltages of each LED in the LED Module is given in Table 1.

Table 1. Luminous flux and forward voltages values for each LED in the LED Module [7]

\begin{tabular}{|c|c|c|c|}
\hline $\begin{array}{c}\text { Emitter } \\
\text { Type }\end{array}$ & Color & $\begin{array}{c}\text { Minimum Luminous } \\
\text { Flux (lm) }\end{array}$ & $\begin{array}{c}\text { Forward } \\
\text { Voltage (V) }\end{array}$ \\
\hline \multirow{4}{*}{ RTBW } & Red & 39.4 & 2.1 \\
\cline { 2 - 4 } & True Green & 70 & 3.4 \\
\cline { 2 - 4 } & Blue & 13.8 & 3.5 \\
\cline { 2 - 4 } & Cool White & 90 & 3.3 \\
\hline
\end{tabular}

* At the forward current $I=350 \mathrm{~mA}$, and the junction temperature $T=25^{\circ} \mathrm{C}$; RTBW: Red, True green, Blue, White

The spectrum of the colors of the LEDs in the module are given in Figure 4.

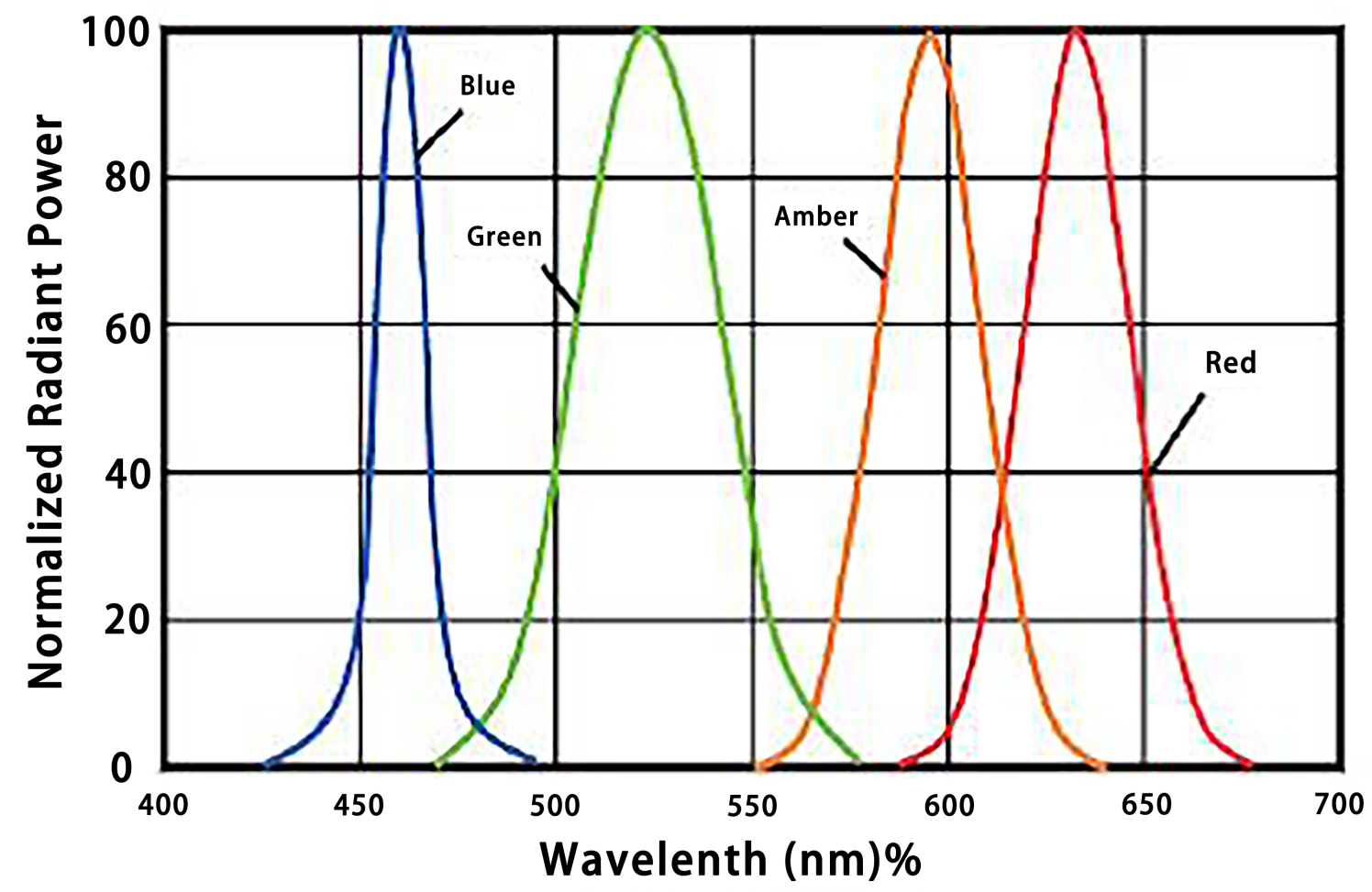

Figure 4. Color spectrum for the LEDs in the module $T=25^{\circ} \mathrm{C}$ [7] 


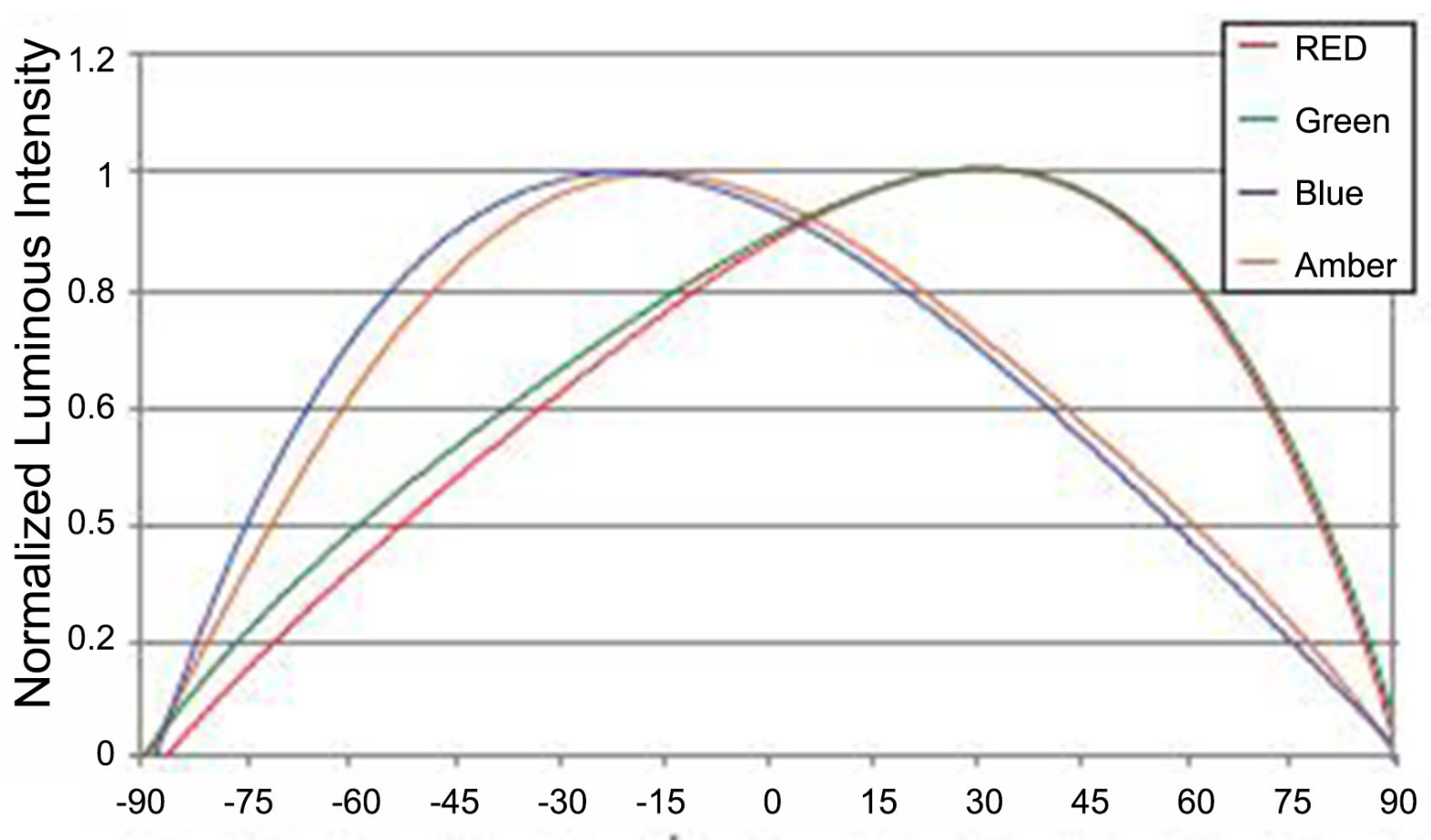

Figure 5. The radiation diagram for the LEDs of different colors in the chip [7]

As seen, the colors are not very pure for green and red LEDs, that is, their spectral bandwith at half intensity $\left(\Delta \lambda_{0.5}=\lambda_{t 05}-\lambda_{-0.5}\right.$ is about $60 \mathrm{~nm}$ and $40 \mathrm{~nm}$, respectively) is rather broad. However, for the BSSL this is acceptable since as seen from the spectrum their peaks are separated enough to be sensed as different colors by the human visual system. The radiation diagram of the LEDs in the chip is given in Figure 5. All the LEDs in the chip have nearly $95 \%$ of their luminous intensities at $0^{0}$, and about $90 \%$ at $5^{0}$. Luminous intensity is defined as the luminous flux per unit solid angle (lumen / sr) [8]. Our aim is to effect the subjects at a distance of up to $20 \mathrm{~m}$ from the BSSL. At this distance we need to generate enough illuminance $\left(\mathrm{l}=\mathrm{lm} / \mathrm{m}^{2}\right)$ to have the incapacitation effects on the target human beings but without causing a permanent damage in their eyes. We designed the BSSL for the bright light conditions (photopic vision) where the human eye is most sensitive to the green light at $555 \mathrm{~nm}$. We assume that the BSSL would then properly operate and even more effective for dark conditions (scotopic vision).

In the visible region of light, for a laser of Gaussian beam profile the maximum permissible exposure (MPE) to the laser light that may be incident upon the eye without causing the biological damage is given as $1 \mathrm{~mW}$ by the Laser Institute of America's "Safe Use of Lasers" [9]. This is the average incident power of the visible Gaussian shape laser beam calculated over the area of the limiting aperture of the fully dilated pupil, which is $7 \mathrm{~mm}$, for an accidental exposure to the laser light during an average eye's blinking reflex time of $0.25 \mathrm{~s}$. This average power corresponds to an irradiance level of $2.55 \mathrm{~mW} / \mathrm{cm}^{2}$, or $25.5 \mathrm{~W} / \mathrm{m}^{2}$, on the average area of $0.385 \mathrm{~cm}^{2}$ of the eye for an average human being [9]. For photopic vision conditions this irradiance level corresponds to an illuminance level of at $555 \mathrm{~nm}$.

$$
E_{v}=25.5 \mathrm{~W} / \mathrm{m}^{2} \times 683 \mathrm{~lm} / \mathrm{W}=17416.5 \mathrm{~lm} / \mathrm{m}^{2}=17416.5 \mathrm{~lx}
$$

If the light source is not continuous but in a pulsed train in time, then this value should be multiplied by $n^{-0.25}$, where $n$ is the number of pulses that occur during the period of exposure [9]. If we again assume that the exposure time is the period of the reflex of an average human's eye of $0.25 \mathrm{~s}$, and if we use a pulse train of the frequency of $15 \mathrm{~Hz}$, for this period of time we have $n=15 / 4=3.75$ pulses. Then the maximum illuminance becomes

$$
E_{v} \times n^{-0.25}=17416.5 \times 3.5^{-0.25}=12740.7 l x
$$

To avoid a biological damage the illuminance at the eye of the target human being should not exceed this value. This is rather a big value that is not easily exceeded with a light source if it is not a laser, and if it is not so close.

We considered that the BSSL should be focused to at most a coverage area of $2.4 \mathrm{~m}^{2}$ in the strobing mode at a distance of $20 \mathrm{~m}$ from the BSSL. To shape the beam we tried to use the reflectors at the market but we did not get the desired focusing. Using commercial narrow-angle single lenses available on the market in the flashlights used by the hunters we obtain the desired focusing effect at a range of $20 \mathrm{~m}$. This lens can be used by mounting on the LED chip shown in Figure $2 \mathrm{a}$. By this way we obtain a beam with a beam angle of $5^{\circ}$ as shown in Figure 6.

The solid angle in steradian is calculated for Figure 6 as 


$$
\Omega=\frac{A}{r^{2}}=\frac{2.4}{20^{2}}=0.006 \text { steradians }
$$

The Luminous intensity is calculated for the white colored LED which has the highest luminous flux value $(90 \mathrm{~lm})$ at the given operating conditions.

$$
I_{v}=\frac{\Phi_{v}}{\Omega}=\frac{90}{0.006}=15000 \mathrm{~lm} / \mathrm{sr}
$$

The illuminance it produces across the coverage area is

$$
E_{v}=\frac{I_{v}}{r^{2}}=\frac{15000}{400}=37.5 l x
$$

This value, as expected, is very much lower than the maximum illuminance permissible calculated in (2). Since other LEDs (green, red, blue) in the chip have lower luminous flux values than this value; therefore, it is shown that our BSSL using these LEDs and optical system would never cause a biological damage to the human's eye.

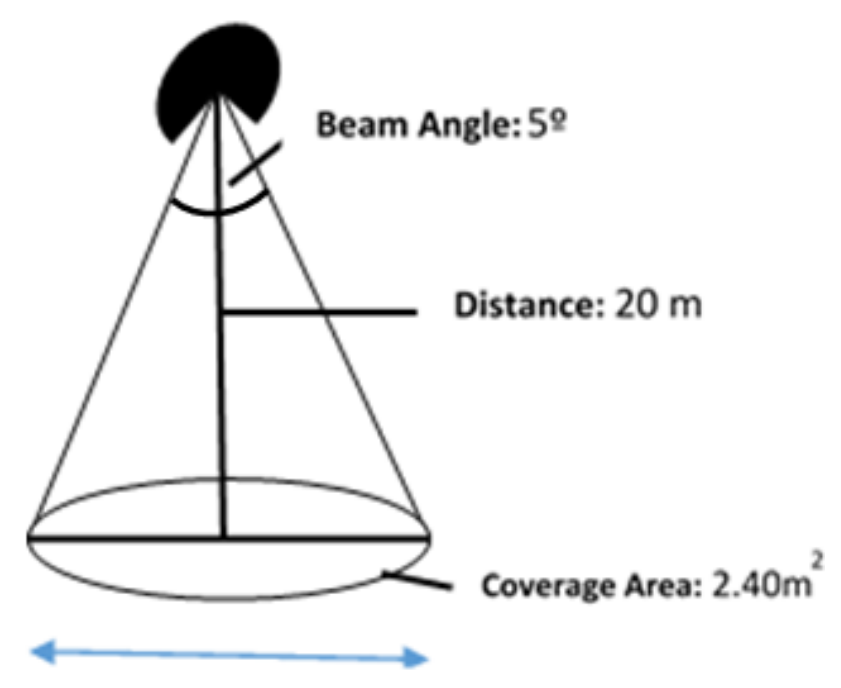

Light Spread: $1.75 \mathrm{~m}$

Figure 6. The BSSL light's focusing geometry at $20 \mathrm{~m}$

To determine whether the illuminance values that these LEDs would generate on the human targets could produce the incapacitating effects should be tested on the humans. We did the testing on ourselves to get the answer to this question. In trying the illuminance levels by using ourselves as samples, we saw that the higher the illuminance levels the more effective the light in affecting the human being. Therefore, by considering the practical reasons such as the size and the weight of the BSSL that can be hand-carried and used easily by security forces we decided that we can use at most three LED chips on the BSSL to increase the illuminance values. When we use three LED chips the flux values given in Table 1 are multiplied by three. These values again will have no biological damage on the human being. This concludes the selection of the LED module of the BSSL.

\subsection{Selection and the Design of the LED Driver}

To drive the LEDs we need a power supply that provides the required average forward current $\left(I_{f}\right)$ to the LEDs at the desired constant voltage. There are two alternatives: either a linear driver or a switch mode power supply (SMPS) could be used [10]. Linear drivers' regulated output voltage is always smaller than the input, and their efficiency increases when the difference between input and output voltage decreases. Their efficiency cannot exceed $80 \%$. Although a linear drivers' footprint is always larger than that of an SMPS, it is good in providing a low noise (low electromagnetic interference, EMI) power. In the SMPS the output current is switched on and off with a frequency up to even $1.5 \mathrm{MHz}$ using MOSFETs as switches. Their efficiencies can exceed $95 \%$. They can provide output voltages lower (buck type), or higher (boost type) than input voltages. Recently there have been many good SMPSs utilizing integrated circuits (ICs) of very small physical dimensions, which include the current and voltage control by arranging the duty times in pulse width modulations, introduced as the LEDs have been increasingly becoming an alternative to the conventional light sources in the lighting applications [3]. Also there are a lot of commercial SMPS drivers have become available on the market with very reasonable prices. For the BSSL we decided to use commercial buck type SMPS driver due to its small size $(4.2 \mathrm{~cm} \times 3.2 \mathrm{~cm} \times 1.2 \mathrm{~cm})$, large input voltage range $(9-30 \mathrm{~V})$, low cost and good efficiency (up to $93 \%$ ) to drive the LED module. The SMPS we selected is a no-name, inexpensive three-channel LED driver, including three separate HV9910 ICs for each channel [11].

We have 3 LED chips, each has 4 LEDs of white, blue, green and red colors. We decided to drive each group of 3 LEDs of the same color with a dedicated SMPS driver. Therefore, we needed 4 LED drivers to drive 4 LED channels of the same color. Hence, we used 2 SMPS drivers in the BSSL, having 2 spare channels left unused. Dedicating a separate driver to a color is required because each LED of different color needs a different forward voltage, $V_{f}$, for the same amount of the forward current, $I_{f}$, as given in the data sheet [7]. We decided to connect the three LEDs of the same color in different chips in series. Therefore, they will have the same forward current. The voltage across these serially connected three LEDs of the same color becomes $3 \times V_{f}$. The design of the circuit is given in Figure 7. 


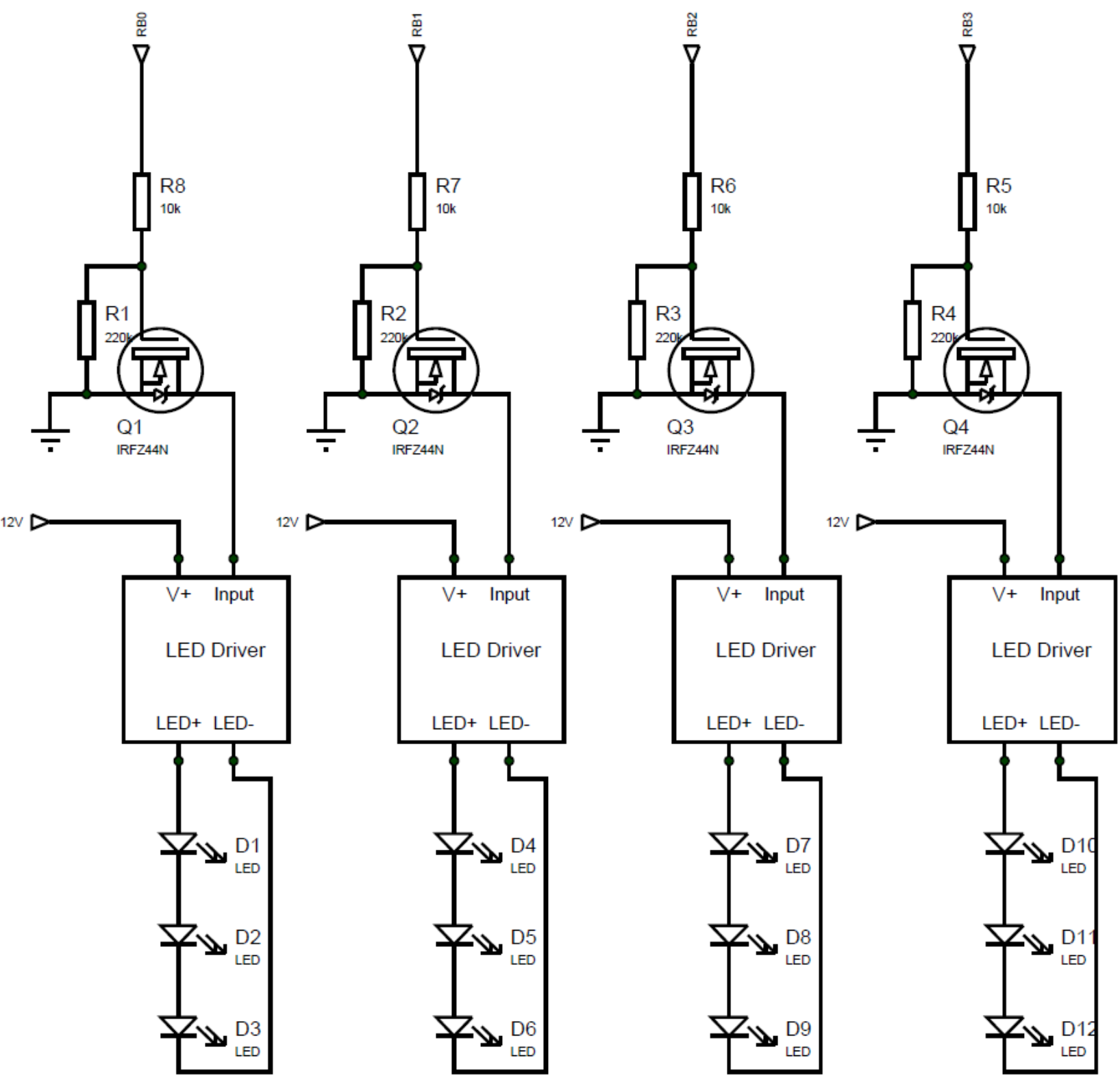

Figure 7. Switching circuit of the BSSL's Switching Unit, each driver, also switched by the MOSFETs to obtain light patterns, provides the current to the individual LEDs of red, green, blue and white color on 3 different chips.

If we take the LEDs forward voltages from Table 1 then serially connected 3 LEDs' forward voltages, the total luminous fluxes, illuminances they produce at $20 \mathrm{~m}$, and the power consumed for each color are given in Table 2.

Table 2. Luminous fluxes, illuminances, total forward voltages and the total power consumption of 3 LEDs of the same color connected in series [7]

\begin{tabular}{|c|c|c|c|c|}
\hline $\begin{array}{c}\text { Number } \\
\text { and Color } \\
\text { of the } \\
\text { LEDs }\end{array}$ & $\begin{array}{c}\text { Minimum } \\
\text { Luminous } \\
\text { Flux (lm) }\end{array}$ & $\begin{array}{c}\text { Illuminance } \\
\text { at 20m (lx) }\end{array}$ & $\begin{array}{c}\text { Forward } \\
\text { Voltage } \\
(\mathrm{V})\end{array}$ & $\begin{array}{c}\text { Power } \\
\text { consumed } \\
(\mathrm{W})\end{array}$ \\
\hline $3 \times$ Red & 118.2 & 49.2 & 6.3 & 2.2 \\
\hline $\begin{array}{c}3 \times \text { True } \\
\text { Green }\end{array}$ & 210 & 87.6 & 10.2 & 3.6 \\
\hline $3 \times$ Blue & 41.4 & 17.3 & 10.5 & 3.7 \\
\hline $\begin{array}{c}3 \times \text { Cool } \\
\text { White }\end{array}$ & 270 & 112.5 & 9.9 & 3.5 \\
\hline
\end{tabular}

$$
I_{f}=350 \mathrm{~mA}, \text { and } T_{j}=25^{\circ} \mathrm{C}
$$

The LED's output optical power, the luminous flux, is linearly proportional to the LED's forward current, $I_{f}$, as

$$
P_{o}=\eta_{e x} \cdot h . v . \frac{I_{f}}{q}
$$

where $\eta_{e x}$ is the external quantum efficiency of the semiconductor material in producing the optical photons from the recombined charge carriers, $h$ is the Planck's constant, $v$ is the frequency of the photon, and $q$ is the electronic charge [12]. However, this equation holds up to a certain level of forward current, beyond which the curve levels off due to the heat generated by the recombination of current carriers within the junction area of the LED. The 
luminous flux decreases also linearly with the junction temperature increase. The increasing forward current increases the junction temperature and then this decreases the luminous flux. The forward voltage of a LED slightly increases with the increase in the forward current, and decreases slightly with temperature increase. Although the LEDs can be operated at higher current levels to provide higher radiant powers, we did not choose higher current values in order to avoid heating in the LED module. However, if a cooling system is used to keep the junction temperature constant, it is easy to increase the current level on the SMPS by arranging the duty cycle.

\subsection{The Power Source}

For the four channels we need a total current $350 \mathrm{~mA} \times 4=1400 \mathrm{~mA}$. For the power source of the BSSL we chose 6 re-chargeable lithium-ion batteries, each of which has $3.7 \mathrm{~V}-3200 \mathrm{~mA}$ capacity. To provide a voltage greater than the highest voltage needed $(10.5 \mathrm{~V})$ we connect 3 batteries in series and then connect these in parallel with the other 3 batteries to obtain $11.1 \mathrm{~V}$ with $6400 \mathrm{~mA}$ current. This power source is connected to the 4 channels of the 2 SMPSs to provide nearly for a 4.6 hours of operation for the BSSL in one charge cycle.

\subsection{The Electronic Control Unit (ECU)}

The block schematic of the ECU designed is shown in Figure 8. The ECU allows us to generate the temporal and spectral changes of the light pulses depending on the user 'mode' choice, namely, the flashlight mode (as a lantern) and the strobing mode (as an incapacitator). After the user selects the mode, the 'Signal Circuit' transmits the light in the desired synchronicity of the selected mode to the 'LED Driver Circuit'. The LED Driver Circuit, in accordance with the signals it receives from the Signal Circuit, generates the voltages and currents as required, and applies them to the LED array.

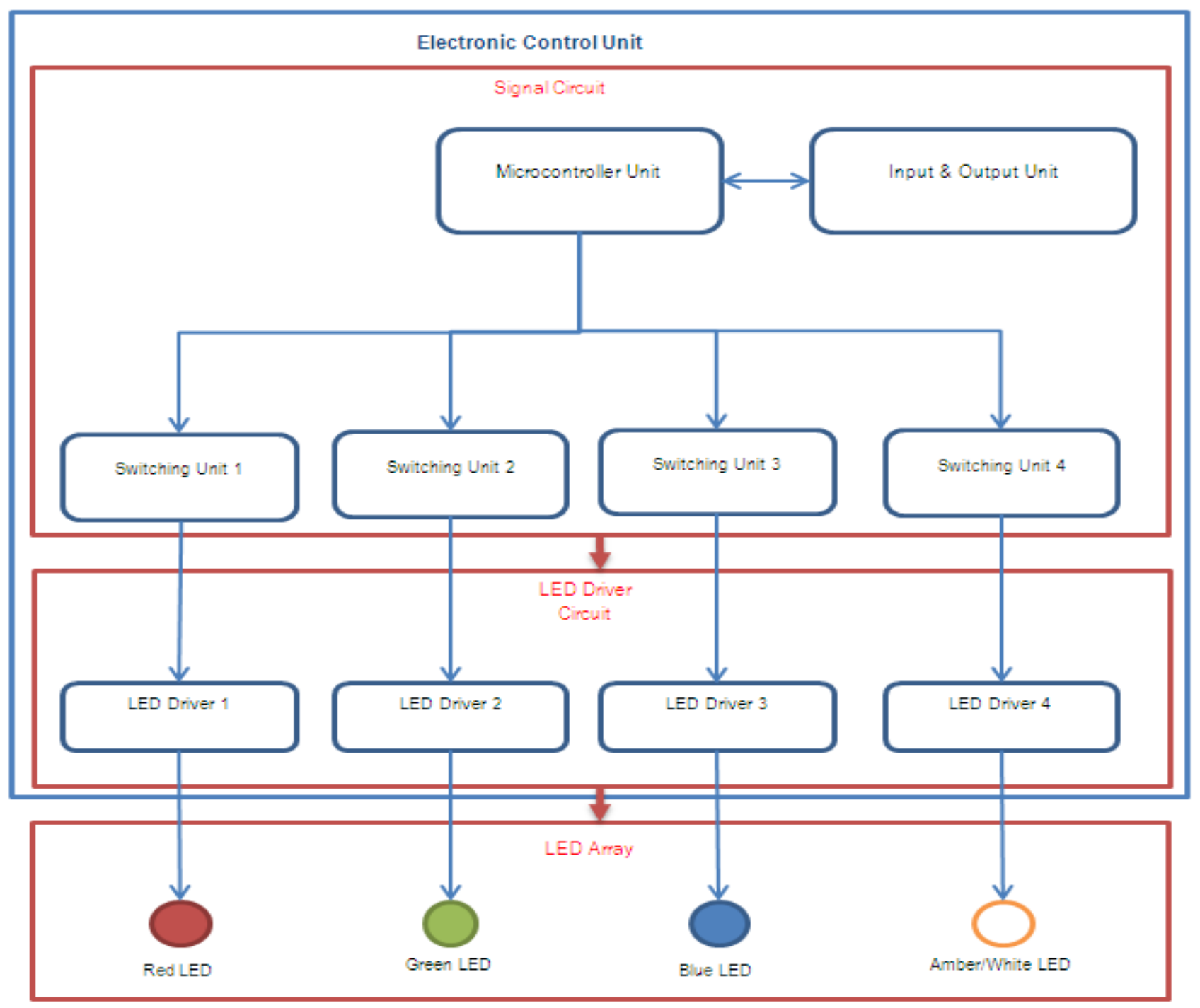

Figure 8. Block schematic of the ECU designed 
The signal circuits used to generate the signals required by means of an embedded software running in the micro controller (16F877A) [13]. The light pulse patterns are generated to change the light beam from the BSSL temporally and spectrally in accordance with a pattern desired. In the microcontroller unit design, PIC 16F877A microcontroller from Microchip was used together with $20 \mathrm{MHz}$ crystal oscillator. The PIC makes it possible for us to control the change of the desired frequency simultaneously with 4 different LED arrays and in 4 different frequency synchronicities. In order to use more than one LED array simultaneously analog signal inputs should be generated and applied to the LED drivers that drive the 4 channels of LED arrays. Also, since 4 output ports were used for driving each LED array, the separate 4 output ports available in PIC 16F877A are useful.

The Switching Unit we designed is shown in Figure 7. As seen, the 4 n-channel MOSFETs (IRFZ44N) [14], drive the individual LED drivers with the signal applied from the microcontroller circuit. The LED drivers have their own switching MOSFETs to generate the PWM signals required with much higher frequencies than those generated by the microcontroller. Our light patterns are generated by the microcontroller within the range of frequencies less than $25 \mathrm{~Hz}$. The patterns of light are applied to the LED arrays by means of the 4 MOSFET switches (Q1, Q2, Q3, and Q4) for each colors. As shown in Figure 7, these patterns are then generated on the LED arrays by the individual LED drivers, each for one color on each LED chip. Each LED driver drives 3 LED chips connected in series.

We designed and developed the embedded software required that enables us to generate the patterns of intensities and colors of the light pulses provided by the LEDs. The software is loaded into PIC 16F877A microcontroller that provides the management of the signal unit, in accordance with the patterns defined by the embedded software. For the software code development, $\mathrm{C}$ programming language was used for its flexibility and commonality. In order to convert the developed software code to the machine language, a CCS $\mathrm{C}$ compiler was used.

\section{Results}

Table 3. The calculated and measured photometric values of the BSSL in the flashlight mode

\begin{tabular}{|c|c|c|c|c|}
\hline & \multicolumn{2}{|c|}{ Calculated } & \multicolumn{2}{c|}{ Measured } \\
\hline $\begin{array}{c}\text { Number } \\
\text { and Color } \\
\text { of the } \\
\text { LEDs }\end{array}$ & $\begin{array}{c}\text { Minimum } \\
\text { Luminous } \\
\text { Flux (lm) }\end{array}$ & $\begin{array}{c}\text { Illuminance } \\
\text { at 20m (lx) }\end{array}$ & $\begin{array}{c}\text { Luminous } \\
\text { Flux (lm) }\end{array}$ & $\begin{array}{c}\text { Illuminance } \\
\text { at 20m (lx) }\end{array}$ \\
\hline 3xRed & 118.2 & 49.2 & 105 & 43.9 \\
\hline $\begin{array}{c}\text { 3xTrue } \\
\text { Green }\end{array}$ & 210 & 87.6 & 186.6 & 78.0 \\
\hline 3xBlue & 41.4 & 17.3 & 41.4 & 17.3 \\
\hline $\begin{array}{c}\text { 3xCool } \\
\text { White }\end{array}$ & 270 & 112.5 & 242 & 101.2 \\
\hline
\end{tabular}

Measurements were taken in flashlight mode and strobing mode with the latest BSSL test version. The Signal Test Unit was run in flashlight mode and the effects of the lights in different intensity on the human vision were tested. The photometric quantities calculated and measured by using an Everfine GO-2000 Goniophotometer are given for each color in flashlight mode in Table 3.

As seen from Table 3, there is a reasonable agreement between the calculated and measured values. We believe that the differences between them are originating from the factors that: the spatial distributions of the beams generated simultaneously from the three chips are not coinciding $100 \%$, and therefore, simply adding their luminous fluxes linearly introduces some error; the measurements were performed not in a temperature controlled room, and there were some tolerances in the geometrical arrangements during the measurements; the individual LEDs we used may have some tolerances with the values given in the datasheets provided by the manufacturer. However, the agreement to this extent verifies that our design is correct within these tolerances.

Thermal tests of the BSSL were performed with a FLIR E-60 thermal camera that measured the heat emanating from the BSSL for 5 hours at the ambient temperature of $25^{\circ} \mathrm{C}$. The results of the measurements showed us that the temperature in the drivers did not exceed $26.6^{\circ} \mathrm{C}$. Therefore, the BSSL can be operated at the selected forward current of $350 \mathrm{~mA}$ continuously, and it does not need a cooling system.

\subsection{Generating the Light Patterns}

The light patterns to incapacitate the human being are generated in the strobing mode of the BSSL. The current generated by the buck type LED drivers is switched on and off at $500 \mathrm{kHz}$. The precise average forward current of $350 \mathrm{~mA}$ flowing through the LEDs is set by arranging the duty cycle in the pulse width modulation (PWM) on the current pulses. The light patterns from the LEDs to incapacitate the human being should mostly be in the frequency range less than $25 \mathrm{~Hz}$, where our eyes can detect the flickering. These patterns of the light are generated on the high-frequency carrier with an on-off keying (OOK) modulation. The OOK modulation on the carrier signal generated by the LED drivers is accomplished by the MOSFET switching circuits given in Figure 7. The patterns of light pulses in time (temporal) and color (spectral) are arranged by the embedded software, then the signals to modulate the light pulses are formed by the PIC in accordance with the pattern defined in the software, and these signals are applied to the gates of the MOSFET switches that drive the LEDs to modulate the carrier signal accordingly.

\subsection{Human under the Effects of Light Pulses}

A very powerful light as a beam of a laser or an incoherent light from a very powerful light source can be directed to a 
human subject to make him turn away the light source or close his/her eyes. This gives enough time for the security forces to catch the subject and take her under control. Although non-lethal, such a weapon may cause permanent damages on the subject's eyes. We are not working on such a weapon in this study, but we are trying to design a system to incapacitate a human being by directing her a time and color changing light pulses. It is non-lethal, and does not give a permanent damage to human's eyes. To achieve and demonstrate this hypothesis we designed the BSSL, a LED incapacitator, to generate the light pulses required, and tested.

In human the brain forms vision through the vision channel and regulates the biological effects in the human body through a non-visual channel [1]. When the light pulses enter into the eye, the rod and cone-shaped photoreceptors in the retina transform the light signals into biological signals that travel through the optic nerve to the sympathetic nerve of the thalamus, and to the back of the brain cortical visual area, which produces the vision [15]. The human non-visual mechanism, on the other hand transmits the signals to the hypothalamic suprachiasmatic nucleus (SCN) by different photoreceptors, and transmits them to the pineal gland, which controls and regulates the physiological parameters of rhythm and other biological effects [1].

How the light pulses of the same color at certain frequency affect the vision and the biological functions of a human being? There are four kinds of brain waves: alpha, beta, theta and delta waves [1], [4], [16], [17]. When the brain is in relaxed mode the brain is emitting alpha brain waves of frequency $8-12 \mathrm{~Hz}$. As seen in Figure 9, people are more sensitive to the flickering effects around $8-12 \mathrm{~Hz}$, where the frequency of brain's bioelectrical signal through the eyes closes to the brain wave frequency [1], [15]. So, if subjected to a light flickering at these frequencies a human being could experience nausea, vomiting, disorientation, and temporary blindness, at the same time may be accompanied by headache and even vertigo. Stimulation by light flickering at alpha frequencies, coinciding with the dominating bandwidth of the electroencephalographic (EEG) signal, can induce an electrical brain rhythm effect that in turn could enhance the neuronal activity in the brain. This will lead to the increase of regional cerebral blood flow and cerebral blood volume to increase intracranial pressure which could cause the effects to occur [1], [18], [19], [20]. The frequency of our brain waves starts to get synchronized with this modulation as a result of the light stimulation. This is called "photic brainwave entrainment" [18]. As a result of this synchronization, the symptoms of photosensitivity may be seen. Photosensitivity is the response of abnormal visual sensitivity given by the brain to flashes, intermittent light sources or more complex impulses such as television-video games and visual patterns [19].

Studies have also shown that the majority of people that are susceptible to the change of intensity of light at some frequencies $(15-70 \mathrm{~Hz})$ may have such symptoms of dizziness, nausea and the like. Studies also showed epileptic symptoms of people at $15 \mathrm{~Hz}$ rate with over 90 seconds of continuous staring at a strobe light [16].

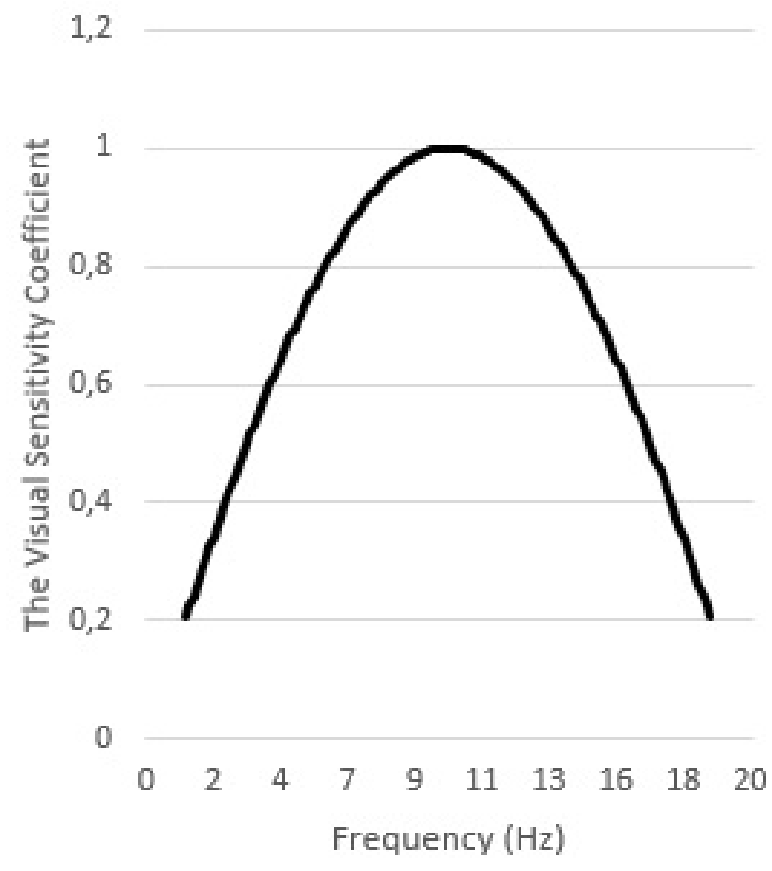

Figure 9. Visual sensitivity curve for the human being [1]

How the change in color of the light pulses in time affects the vision and the biological functions of a human being? The retina in the human eye has many receptors, of which the receptors in conical shape have strong spectral sensitivity. The cone receptors are also divided into types that sense different wavelength of color in light. They mainly sense the photopigments of principal colors of red, green, and blue to encode light [15]. $64 \%$ of these cells are sensing red color at the maximum level at $664 \mathrm{~nm}, 32 \%$ green light at $533 \mathrm{~nm}$, and $2 \%$ blue light at $437 \mathrm{~nm}$ [1]. When different colors of pulse of light in red, green and blue which stimulate the eye maximum are coming at a certain frequency are applied to the eye of the human being the three kinds of the cone cells transform these into electrical signals. Some amount of these signals arrive to pineal through the non-visual channel. The pineal can secrete and synthesize biological glue and peptide substances, which can regulate the nervous secretion and reproductive system function [1]. There is also observed biochemical changes in the brain causing a change in the cerebrospinal fluid concentration of neurotransmitters which can cause emotional out of control, increased heart rate, blurred vision, muscle cramps, nausea, vomiting, and other physiological and psychological effects [1]. At the same time, different colors of light can cause effects in the brain's internal biological activities, some of which can induce brain ischemia, inability to produce visual signals, and temporary blindness [1], [21]. The red color at the wavelength of $660-720 \mathrm{~nm}$ has a high risk of creating crisis depending on blue and white colors. After 685 children who watched the cartoon called Pokemon in 1997 in Japan 
saw the red and blue lights flashing in the rocket launching scene, they applied to the emergency with attacks and after this incident the role of the colors in the human photosensitivity started to be questioned again [19].

Flash durations, flash colors and the effects of rapidly changing frequencies within the alpha-theta band have been, and are still being, investigated for their effects on brain activity. The general rule of light-brain interaction from the frequency-following effect is that all three factors play an important role in modulating brain rhythms. As these factors become more variable and more random, they introduce more modulation, and thus more confusion in the brain rhythms [5].

\subsection{Testing of Effects of Temporal and Spectral Change of Light on Human}

We tested the light pulses generated by the BSSL on ourselves. We are planning to use in the future a number of human subjects for further testing to verify the efficiency of the incapacitating effects of the light pulses and provide statistical data about the effects generated by the light pulses from the LED incapacitator (the BSSL).

In our search for the most suitable pattern of light to obtain incapacitation effects we changed both the exposure time (i.e. duration of the radiation pulses), and their rate of repetitions, and we used combinations of colors in the flashing lights. The light pulses in different colors selected randomly were used in different frequency ranges and the effects on ourselves were observed.

In the tests, as expected, we observed that red light is more effective in terms of affecting our visions compared to the other colors, and also its effects lasted longer than those of the other colors. Furthermore, we observed that since the white light provides more luminous intensity than the other colors, it causes short-term visual impairment (afterimage).

The tests are performed including only red, only green, only blue and only white at the range of $7-15 \mathrm{~Hz}$ frequency. Here we mean by "the frequency" is the inverse of the period between the bursts of the light pulses in the pattern. After testing various patterns of light pulses we identified the most effective pattern among the patterns we generated and tested. These light pulse patterns are modulating the carrier frequency $(500 \mathrm{kHz})$ at 7,10 , and $15 \mathrm{~Hz}$. Figure 10 shows the most effective light pulse patterns as we determined during the tests.

We experienced some physiological effects such as temporary visual impairment and loss in balance for a few minutes when we were susceptible to the patterns in Figure 10. The duration of these effects varied. The most effective pattern observed was for $15 \mathrm{~Hz}$ confirming with the visual sensitivity curve of Figure 9. As explained in the previous section this frequency coincides with the EEG band also. We did not experience any blackout during the tests. The patterns that have frequencies lower than $7 \mathrm{~Hz}$, and higher than $20 \mathrm{~Hz}$ were not effective. The higher the intensity of the pulses the higher the effects observed.

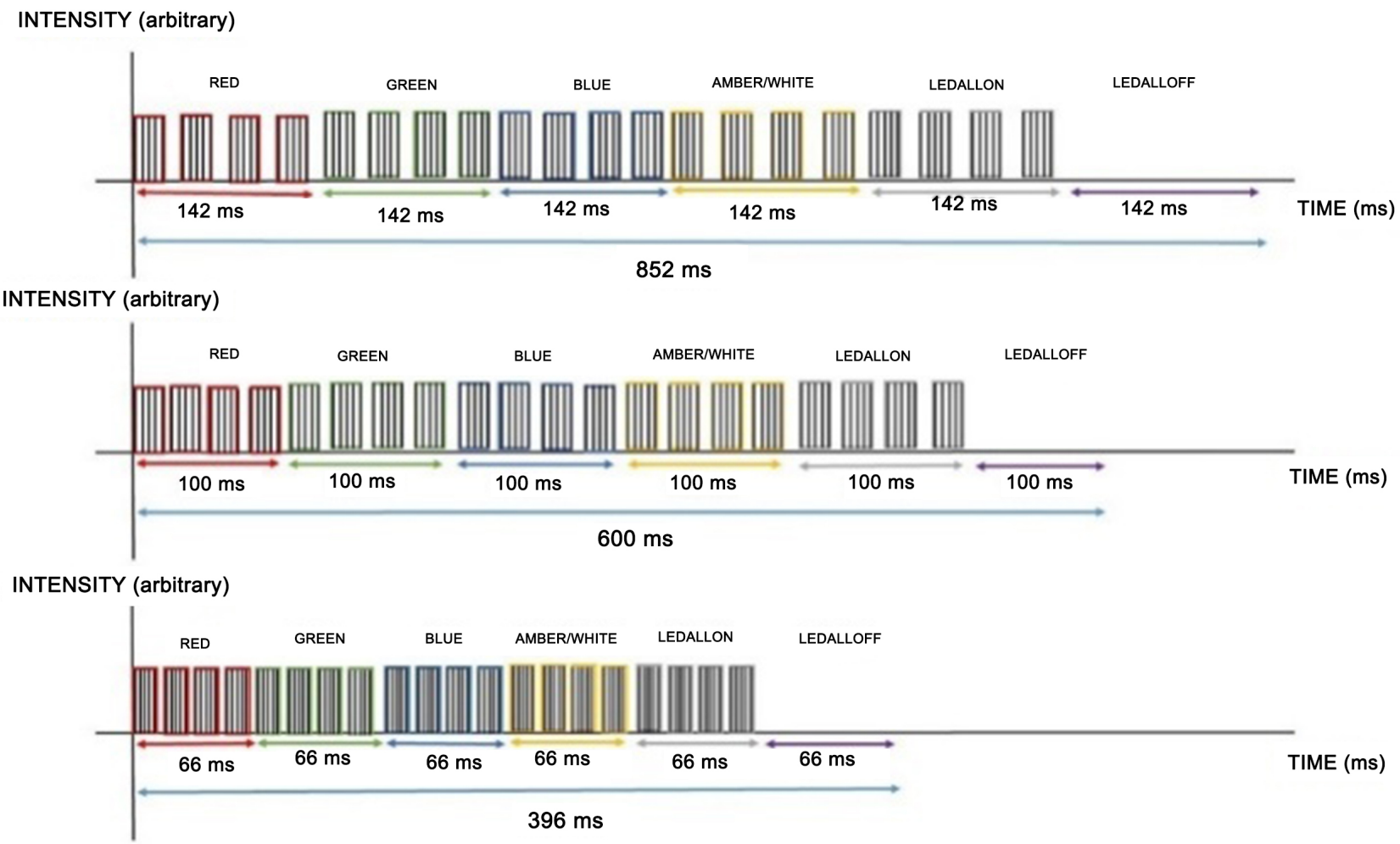

Figure 10. The most effective light pulse patterns in our tests. The pulses are actually OOK modulated carrier of $500 \mathrm{kHz}$. 


\section{Conclusions}

We have designed and developed a non-lethal incapacitator using light emitting diodes (LEDs) to be used by the security officers. We named the LED incapacitator developed as the blocker security system with LEDs (BSSL). Our objective in developing the BSSL is to test the hypothesis if the light pulses of varying intensity and color in time can cause incapacitation on human targets without causing permanent eye damages. We gave the details on how such an integrated lamp is designed by using the high-power LEDs on chips, the switched mode power supplies as LED drivers. The BSSL we designed has three LED chips, each having four LEDs of red, green, blue and white colors. It has two modes of operations the flashlight mode (as a lantern) and the strobing mode (as an LED incapacitator). The patterns of light pulses of frequencies $7-15 \mathrm{~Hz}$ with varying colors are generated by using an embedded software that runs in a PIC, which generates the required pulses of voltages that modulate the PWM signals from the LED drivers. This modulation produces the light patterns as desired. We measured the illuminance values that the BSSL generates at the maximum range of $20 \mathrm{~m}$ and verified that they are confirming the design. The light pulses in the range of $7-15 \mathrm{~Hz}$ are coinciding with brain's alpha and beta waves and also falling within the band of EEP signals. In the literature it is claimed, and sometimes in part shown that light pulses of red, green, and blue colors are changing in time at these frequencies can have some incapacitating effects on human beings such as nausea, dizzying, blackout, vomiting, and even vertigo. By creating light patterns of these frequencies and randomly changing colors through the BSSL we tested the effects on ourselves. Within this limited sample size of two, we observed that indeed the effective frequencies are in the range of $7-15 \mathrm{~Hz}$, the most effective being at $15 \mathrm{~Hz}$. The red light was the most effective among other colors. The higher the intensity of the light pulses the more effective the particular pattern. We partly verified the hypothesis that the light pulses of varying frequency and colors have incapacitating effects on human being. We experienced some physiological effects such as temporary visual impairment and loss in balance for a few minutes when we were susceptible to the patterns in Figure 10. This research on LED incapacitator on this scale and detail may be the first in the literature to our knowledge. Future work will aim to investigate the effects of light pulses on the visual system and the biological functions on the humans in more detail using a large size of subjects in the tests.

\section{Acknowledgements}

The authors thank to Çankaya University, and Ministry of Science, Industry and Technology of Turkey (KOSGEB) for supporting this research.

\section{REFERENCES}

[1] Daiqiang Wei, Renjun Zhan, "Study on the Mechanism of Led Incapacitator Device Based on the Variable Frequency and Variable Waves, progress in Applied Science and Technology", Advanced Materials Research, Vol. 926-930, pp.1183-1186, 2014.

[2] R. Lenk, C. Lenk, "Practical Lighting Design with LEDs", IEEE Press, Wiley, USA, 2011.

[3] Winder S., "Power Supplies for LED Driving", Elsevier Science, Boston, MA, USA, 2008.

[4] Rubtsov V., "Incapacitating flashing light apparatus and method", US7180426 B2, USA, 2007.

[5] Rubtsov V., "LED-based incapacitating apparatus and method", US 7500763 B2, USA, 2009.

[6] EdixeonDx, Ex Series LEDs, “Application Notes", Edison Opto Corporation, USA, 2008.

[7] Edison, Federal 5050 LEDs, Datasheet, Edison-Opto Corporation, 2014.

[8] Alma E.F. Taylor, "Illumination Fundamentals", Lighting Research Center, Renssealer Polytechnic Institute, USA, 2000 .

[9] Laser Institute of America, The IEC-60825-1 and ANSI Z136.1

[10] Abraham I. Pressman, Keith Billings, Taylor Morrey, "Switching Power Supply Design", The McGraw Hill Companies, USA, 2009.

[11] HV9910 IC Data Sheet, Supertex Inc., USA, 2004.

[12] Saleh B. E. A., and Teich M. C., "Fundamentals of Photonics", Wiley- Interscience, Hoboken, NJ, USA, 2007.

[13] PIC 16F87X, Datasheet, Microchip Technology Inc., USA, 2001.

[14] IRFZ44 MOSFET, Datasheet, Philips semiconductors, USA, 1999.

[15] Brin A. Wandell, "Foundations of Vision", Sinauer Associates, Inc., USA, 1995.

[16] Mindalive, https://www.mindalive.com/2_0/ch6.pdf, (Data Download Date: 22.11.2013).

[17] Daab R., "A Brief Introduction to Light and Sound", the Voyager XL User Guide, USA, 1993.

[18] Martel A., "Light Modulation, a new way of looking at lighting", Professional Lighting Design Magazine, vol. 57, 2007.

[19] Baysal L., Bebek N., and Baykan B., "Photosensitivity and Reflex Epilepsies", Epilepsy vol. 20 (Annex 1), pp. 23-31, 2014.

[20] No-Sang Kwak, Klaus-Robert Müller, and Seong-Whan Lee, "A lower limb exoskeleton control system based on steady state visual evoked potentials", Journal of Neural Engineering, Vol.12, pp.1-14, 2015.

[21] Klinghardt D., "The Neurophysiology of Light, The Five Pathways", Journal of Optometric Phototherapy, pp. 35-40, 2003. 